\title{
Whole-exome sequencing identifies variants in invasive pituitary adenomas
}

\author{
XIAOLEI LAN ${ }^{1,2^{*}}$, HUA GAO $^{1 *}$, FEI WANG $^{3}$, JIE FENG $^{1}$, JIWEI BAI $^{1}$, PENG ZHAO $^{4}$, \\ LEI CAO $^{1}$, SONGBAI GUI ${ }^{4}$, LEI GONG $^{1}$ and YAZHUO ZHANG ${ }^{1}$
}

\begin{abstract}
${ }^{1}$ Key Laboratory of Central Nervous System Injury Research, Beijing Neurosurgical Institute, Beijing Tiantan Hospital, Capital Medical University, Beijing 100050; ${ }^{2}$ Department of Neurosurgery, The Affiliated Hospital of Medical College, Qingdao University, Qingdao, Shandong 266071; ${ }^{3}$ Department of Neurosurgery, Provincial Hospital Affiliated to Anhui Medical University, Hefei, Anhui 230032; ${ }^{4}$ Department of Neurosurgery, Beijing Tiantan Hospital, Capital Medical University, Beijing 200050, P.R. China
\end{abstract}

Received March 4, 2015; Accepted March 18, 2016

DOI: $10.3892 / \mathrm{ol} .2016 .5029$

\begin{abstract}
Pituitary adenomas exhibit a wide range of behaviors. The prediction of invasion or malignant behavior in pituitary adenomas remains challenging. The objective of the present study was to identify the genetic abnormalities associated with invasion in sporadic pituitary adenomas. In the present study, the exomes of six invasive pituitary adenomas (IPA) and six non-invasive pituitary adenomas (nIPA) were sequenced by whole-exome sequencing. Variants were confirmed by dideoxynucleotide sequencing, and candidate driver genes were assessed in an additional 28 pituitary adenomas. A total of 15 identified variants were mainly associated with angiogenesis, metabolism, cell cycle phase, cellular component organization, cytoskeleton and biogenesis immune at a cellular level, including 13 variants that occurred as single nucleotide variants and 2 that comprised of insertions. The messenger RNA (mRNA) levels of diffuse panbronchiolitis critical region 1 (DPCR1), KIAA0226, myxovirus (influenza virus) resistance, proline-rich protein BstNI subfamily 3, PR domain containing 2, with ZNF domain, RIZ1 (PRDM2), PR domain containing 8 (PRDM8), SPANX family member N2 (SPANXN2), TRIO and F-actin binding protein and zinc finger protein 717 in IPA specimens were $50 \%$ decreased compared with nIPA specimens. In particular, DPCR1, PRDM2, PRDM8 and SPANXN2 mRNA levels in IPA specimens were approximately four-fold lower compared with nIPA specimens $(\mathrm{P}=0.003,0.007,0.009$ and 0.004 , respectively).
\end{abstract}

Correspondence to: Professor Yazhuo Zhang, Key Laboratory of Central Nervous System Injury Research, Beijing Neurosurgical Institute, Beijing Tiantan Hospital, Capital Medical University, 6 Tiantan Xili, Beijing 100050, P.R. China

E-mail: zyz2004520@yeah.net

${ }^{*}$ Contributed equally

Key words: pituitary adenomas, exome sequencing, invasive, variant, PR domain family
By contrast, the mRNA levels of dentin sialophospho protein, EGF like domain, multiple 7 (EGFL7), low density lipoprotein receptor-related protein $1 \mathrm{~B}$ and dynein, axonemal, assembly factor 1 (LRRC50) were increased in IPA compared with nIPA specimens ( $\mathrm{P}=0.041,0.037,0.022$ and 0.013 , respectively). Furthermore, decreased PRDM2 expression was associated with tumor recurrence. The findings of the present study indicate that DPCR1, EGFL7, the PRDM family and LRRC50 in pituitary adenomas are modifiers of tumorigenesis, and most likely contribute to the development of oncocytic change and to the invasive tumor phenotype.

\section{Introduction}

Pituitary adenomas account for $10-15 \%$ of all intracranial neoplasms and are incidentally identified in $<27 \%$ of non-selected autopsies (1). The clinical presentation of pituitary adenomas depends on the structural and functional characteristics of the tumor (2). The World Health Organization (WHO) categorizes pituitary tumors into typical adenomas, atypical adenomas and pituitary carcinomas; of which, typical adenomas constitute the major class. However, the WHO classification does not offer an accurate association between the histopathological findings and the clinical behavior of the tumor (3). An estimated 35-55\% of pituitary adenomas demonstrate invasion into bones, dura or adjacent structures, including the cavernous or sphenoid sinuses (4). Clinically defined invasive pituitary adenomas (IPAs) demonstrate earlier and more frequent recurrences, and may be resistant to conventional treatments, such as surgery and radiotherapy (5). Specific biomarkers that distinguish between aggressive and nonaggressive pituitary adenomas have not yet been identified, although certain studies suggest that the Ki-67 proliferation index may be of diagnostic value (3). The WHO classification of endocrine tumors indicates that invasion of the surrounding structures, size at presentation, an elevated mitotic index, a Ki-67 labeling index of $>3 \%$ and extensive tumor protein p53 (p53) expression are indicators of aggressive behavior (6,7). However, Ki-67 and p53 labeling index evaluations demonstrate subjective variability, and the cutoff values 
are controversial (8). Clinically, endocrine tumors present a challenging management problem, with a high frequency of incomplete resections, tendency for recurrence and notable morbidity (9).

Previously, several studies attempted to identify novel molecular markers [fibroblast growth factor receptor 4, matrix metalloproteinases, Ki-67, p53, cyclooxygenase-2, galectin-3, angiogenesis molecules and pituitary tumor-transforming 1 (PTTG)] that require additional validation (10-13). In a previous study, multivariate Cox regression analysis assessed galectin-3 immunohistochemical expression in $\geq 30 \%$ of neoplastic cells; galectin-3 messenger RNA expression was indicated to be a strong predictive factor of recurrence or tumor progression $(\mathrm{P}<0.001)$; and a Ki-67 labeling index of $>3 \%(\mathrm{P}=0.019)$ was indicated in the 81 cases with available follow-up data (12). PTTG expression may be associated with tumor invasiveness and microvessel density of pituitary adenomas (13). Apoptosis and mitoses represent two adverse and asynchronous events that maintain the optimal cell numbers; cytogenetic analysis may, therefore, be useful in defining the biological invasion of pituitary tumors (14). In addition, predicting the subsequent risk of disease invasion or drug sensitivity is challenging. However, mutations in classic oncogenes and tumor-suppressor genes are rarely associated with these tumors (3-6,8-15). Nonfunctioning pituitary adenomas (NFPAs) result in few somatic mutations, which is consistent with the associated low proliferation rates and benign nature; however, mechanisms other than somatic mutation are likely to be involved in the etiology of sporadic NFPAs (16). The majority mechanisms of endocrine tumorigenesis differ significantly from those associated with haematological malignancies and non-endocrine tumors (17). In addition, the genetic events underpinning the development of invasion or refractory pituitary adenomas are not yet understood (18).

In order to identify the genetic events that may be contributing to the invasion of pituitary adenomas, whole-exome sequencing, which has been successfully used to find variants in multiple tumor types, was applied $(16,19-21)$. Through stringent variant calling and filtering parameters, 15 identified variants were mainly associated with cell cycle phase, cellular component organization and biogenesis at cellular level by whole-exome sequencing in combination with homozygosity mapping between IPAs and non-invasive pituitary adenomas (nIPA). The present study supports the role of somatic variants of the PR domain (PRDM) gene family, which is known to control cell proliferation in cancer and in normal development, in IPAs.

\section{Materials and methods}

Patients and specimens. Specimens from six IPAs and six nIPAs were obtained from patients that underwent endoscopic transsphenoidal surgery between December 2009 and January 2010 at Beijing Tiantan Hospital, Beijing, China. Informed consent was obtained from all individuals and ethical approval was obtained form the Institutional Review Board of Beijing Tiantan Hospital Affiliated to Capital Medical University. Pituitary adenomas, obtained from 12 patients ( 5 men and 7 women; mean age, 40.7 years; range, 16.0-63.0 years) that did not have a family history of endocrine neoplasia, were characterized based on presurgical clinical and biochemical findings, including a pituitary hormone test. This tested for 12 types of pituitary hormone: Growth hormone, adrenocorticotropic hormone, follicle-stimulating hormone, luteinizing hormone, estradiol, progesterone, human growth hormone, cortisol, total triiodothyronine, total thyroxine, thyroid-stimulating hormone and prolactin (PRL) levels (4 patients in normal range, 8 patients with increased PRL levels; normal range, $2.5-17 \mathrm{ng} / \mathrm{ml}$ ). Pituitary adenomas were also characterized based on morphological and immunohistochemical analysis of removed tissue samples (Table I). Cases with multiple hormonal changes according to the clinical and pathological data were excluded. The following IPA diagnostic criteria were adopted: i) Knosp classification grade III-IV tumors and Hardy classification invasive adenomas; ii) tumor cells confirmed via pathology as invading the sphenoid bone or adjacent dura mater; iii) tumor cells invading the sphenoid sinus cavity or peripheral vascular and nerve; iv) Ki-67 labeling index of $>3 \%$ (22). The tumors did not have atypical features, and constituted the 'discovery' set of tumors for exome capture and DNA sequence analysis. An additional 28 pituitary adenomas, histologically confirmed, were obtained from 13 women and 15 men (mean age, 61 years; range, 17-71 years), and these constituted the 'validation' set. For histological analysis, the tumor specimens were divided into two sections. One was stored in liquid nitrogen and the other was fixed in $4 \%$ paraformaldehyde for $24 \mathrm{~h}$ (Sinopharm Chemical Reagent Beijing Co., Ltd., Beijing, China) within 0.5 h of surgery. After washing for $6 \mathrm{~h}$ in flowing water, the specimens underwent gradient dehydration in alcohol, were embedded in paraffin wax (Leica Biosystems Richmond Inc., Richmond, IL, USA) and sectioned at a thickness of $5 \mu \mathrm{m}$. Sections were incubated with primary mouse anti-human monoclonal PRDM2 antibody (catalog no., ab3791; dilution, 1:200; Abcam, Cambridge, MA, USA) at $4^{\circ} \mathrm{C}$ overnight. Next, sections were washed three times with phosphate-buffered saline (PBS; ZSGB-BIO), then incubated with DyLight-conjugated AffiniPure secondary antibody (goat anti-mouse IgG H+L; catalog no., ZF-0313; ZSGB-BIO, Beijing, China) with fluorescence was added at room temperature for $1 \mathrm{~h}$ followed by 3 washes with PBS (5 min each). Streptavidin-Biotin Complex (ZSGB-BIO) was added for $20 \mathrm{~min}$ and then the sections were washed with PBS. Next, sections were mounted with ProLong Gold Antifade reagent (ZSGB-BIO) with DAPI (Invitrogen; Thermo Fisher Scientific, Inc., Waltham, MA USA). Staining was visualized using a LEICA-TCS-SP5II microscope (Leica, Wetzlar, Germany). The percentage of DAPI-stained cells exhibiting PRDM2 immunoreactivity was analyzed in 5 randomly selected high power fields.

Specimen preparation, exome capture, DNA sequencing and bioinformatics analysis. Total DNA was extracted from pituitary adenomas using the QIAamp DNA Mini Kit (Qiagen GmbH, Hilden, Germany). An aliquot containing $5 \mu \mathrm{g}$ of genomic DNA was purified and quantified from each specimen. Exome enrichment was performed by using an ABI SOLiD optimized SureSelect Human All Exon kit (Agilent Technologies, Inc., Santa Clara, CA, USA), which included the exonic sequences of $\sim 18,000$ genes, covering a total of $42 \mathrm{Mb}$ of genomic sequences. The enriched exome libraries were then 
Table I. Clinical data of patients.

\begin{tabular}{|c|c|c|c|c|c|c|c|c|c|}
\hline Specimen & $\begin{array}{c}\text { Tumor } \\
\text { sub-type }\end{array}$ & $\begin{array}{l}\text { PRL, } \\
\mathrm{ng} / \mathrm{ml}\end{array}$ & $\begin{array}{l}\text { Age, } \\
\text { years }\end{array}$ & Gender & $\begin{array}{c}\text { Tumor } \\
\text { volume, } \mathrm{cm}^{3}\end{array}$ & Histology & $\begin{array}{c}\mathrm{Ki}-67 \\
\text { index, \% }\end{array}$ & Invasive & $\begin{array}{c}\text { No. of } \\
\text { variants }\end{array}$ \\
\hline 1 & NFPA & 5.8 & 63 & $\mathrm{~F}$ & 16.4 & $(-)$ & $>3$ & Yes & 23 \\
\hline 2 & NFPA & 13.2 & 53 & $\mathrm{~F}$ & 4.8 & $(-)$ & $1-2$ & No & 12 \\
\hline 3 & NFPA & 9.4 & 42 & M & 13.2 & $(-)$ & $>3$ & Yes & 28 \\
\hline 4 & NFPA & 11.7 & 53 & $\mathrm{~F}$ & 7.4 & $(-)$ & $1-2$ & No & 14 \\
\hline 5 & PRL & 182 & 25 & $\mathrm{~F}$ & 10.7 & $\operatorname{PRL}(+)$ & $>3$ & Yes & 27 \\
\hline 6 & PRL & 1,625 & 16 & $\mathrm{~F}$ & 12.2 & PRL (+) & $>3$ & Yes & 37 \\
\hline 7 & PRL & 3,117 & 54 & M & 14.3 & $\operatorname{PRL}(+)$ & $>3$ & Yes & 26 \\
\hline 8 & PRL & 268 & 29 & M & 9.2 & PRL (+) & $>3$ & Yes & 32 \\
\hline 9 & PRL & 123 & 32 & $\mathrm{~F}$ & 2.4 & PRL (+) & $1-2$ & No & 21 \\
\hline 10 & PRL & 233 & 43 & $\mathrm{~F}$ & 3.1 & PRL (+) & $1-2$ & No & 20 \\
\hline 11 & PRL & 2,899 & 34 & M & 1.9 & PRL (+) & $1-2$ & No & 25 \\
\hline 12 & PRL & 2,830 & 44 & M & 4.1 & PRL (+) & $1-2$ & No & 19 \\
\hline
\end{tabular}

PRL normal range, $2.5-17 \mathrm{ng} / \mathrm{ml}$. NFPA, nonfunctioning pituitary adenomas; PRL, prolactin; F, female; M, male.

Table II. Methylation validation primers.

\begin{tabular}{lll}
\hline Gene & \multicolumn{1}{c}{ Forward primer } & Reverse primer \\
\hline PRDM8 & 5'-ATTCCCTTTCAAACGACCAGA-3' & 5'-AAGAGTTGGATACGTCGTAAA-3' \\
PRDM2 & 5'-GGCCAAGAAGCGGAGAACT-3' & 5'-AAGTCACAGCGACTCACCAGC-3' \\
MGAM & 5'-GGCGGAGTCCTTGCTCTTAT-3' & 5'-GTATGACAGTGCAGCTTCAGGA-3' \\
SPANXN2 & 5'-GAGGAGGACGAAGGCCTAGA-3' & 5'-CTCACTACCAATGGCGATGA-3' \\
TRIOBP & 5'-CCAGGCTTCCTCCATGACAC-3' & 5'-TGTGTCCAGCAGGACGATC-3' \\
ZNF717 & 5'-CCTTTCGCTGTAAGTCATTCCT-3' & 5'-TCAGAGAACTCATGCTGGCA-3' \\
PRB3 & 5'-CCCCCACAAGGAGGAAACCA-3' & 5'-CCACAAGGAGGAAACCAGT-3' \\
DPCR1 & 5'-TTCTGATTGGACTCCCTCTC-3' & 5'-TAGTGCGATCTCCTGACCTC-3' \\
DSPP & 5'-ATCTCTTGTAATTTAGCTACC-3' & 5'-AATATATTGGTACATCACCA-3' \\
MX2 & 5'-AGAAGCTTGGACGTGCCAAG-3' & 5'-AGGGGTCCAGGTCACAGCC-3' \\
EGFL7 & 5'-TCCTGGGTTGGGTCAGCCATGC-3' & 5'-AATTGAATGATGTGCAGTTG-3' \\
LRRC50 & 5'-CGAGACCATCCTAGCCAACAC-3' & 5'-TGTTCCTTCTGATGTTCGGAT-3' \\
LRP1B & 5'-AGCCAATTCGAATCCTTGCTA-3' & 5'-TTGCATGACTAATATACCTGTT-3' \\
MAST4 & 5'-CTTGAACTCTGCCTCAAGCATTC-3' & 5'-ACAAGAACTGGTTTGGTAC-3' \\
RP1L1 & 5'-GCTTGCCCTTGATATCCTTTTAT-3' & 5'-TTCATCTGCAAACTTAACTCCG-3' \\
GAPDH & 5'-CAGCTGAGGGACCCATGAA-3' & 5'-AAGTGGTCATTGAGGGCGAT-3' \\
\hline
\end{tabular}

PRDM8, PR domain containing 8; PRDM2, PR domain containing 2, with ZNF domain, RIZ1; MGAM, maltase-glucoamylase ( $\alpha$-glucosidase); SPANXN2, SPANX family member N2; TRIOBP, TRIO and F-actin binding protein; ZNF717, zinc finger protein 717; PRB3, proline-rich protein BstNI subfamily 3; DPCR1, diffuse panbronchiolitis critical region 1; DSPP, dentin sialophospho protein; MX2 myxovirus (influenza virus) resistance; EGFL7, EGF like domain, multiple 7; LRRC50, dynein, axonemal, assembly factor 1; LRP1B, low density lipoprotein receptor-related protein 1B; MAST4, microtubule associated serine/threonine kinase; RP1L1, retinitis pigmentosa 1-like 1; GAPDH, glyceraldehyde 3-phosphate dehydrogenase.

amplified by emulsion polymerase chain reaction (ePCR; Ion PI $^{\text {TM }}$ Hi-Q ${ }^{\text {TM }}$ OT2 200 kit; cat no. a26434), according to the manufacturer's instructions (Thermo Fisher Scientific, Inc.), and based on a library concentration of $0.5 \mathrm{pM}$. The PCR products were then sequenced on a SOLiD5500 sequencer (Thermo Fisher Scientitic, Inc.), and one quadrant of a SOLiD sequencing slide was required for each sample.
Color-space reads were mapped to the hg19 reference human genome using SOLiDBioScope software (5500 W Series Genetic Analyzer V2.0; Thermo Fisher Scientitic, Inc.), which is suitable for a repetitive mapping approach. Single-nucleotide polymorphisms (SNPs) were then called using the diBayes algorithm with conservative default call stringency. Known SNPs available from the Single Nucleotide Polymorphism Database 
Table III. Reverse transcription-quantitative polymerase chain reaction primers.

\begin{tabular}{lll}
\hline Gene & \multicolumn{1}{c}{ Forward primer } & \multicolumn{1}{c}{ Reverse primer } \\
\hline DPCR1 & 5'-AGTGCTGCCTCCTCTTCCTTCTA-3' & 5'-GGGAGCTCTGGAGGTCTTTGTC-3' \\
DSPP & 5'-GCATTTGGGCAGTAGCATGG-3' & 5'-CTGACACATTTGATCTTGCTAGGAG-3' \\
MGAM & 5'-GGCGGAGTCCTTGCTCTTAT-3' & 5'-GTATGACAGTGCAGCTTCAGGA-3' \\
EGFL7 & 5'-ATGTGGATGAATGCAGTGCT-3' & 5'-TGTCCACTCCTGTCGGGTT-3' \\
MX2 & 5'-GCCAGGTGGAGAAAGAGATACACAA-3' & 5'-AGGTCAATGATGGTCAGGTCTGG-3' \\
LRRC50 & 5'-CGAGACCATCCTAGCCAACAC-3' & 5'-TGTTCCTTCTGATGTTCGGAT-3' \\
PRDM2 & 5'-AGCAGCTGCGATTGAGGA-3' & 5'-CAGAGGTGAAATCTGGCTCACTT-3' \\
PRDM8 & 5'-ATTCCCTTTCAAACGACCAGA-3' & 5'-AAGAGTTGGATACGTCGTAAA-3' \\
LRP1B & 5'-AGCCAATTCGAATCCTTGCTA-3' & 5'-TTGCATGACTAATATACCTGTT-3' \\
RP1L1 & 5'-AGAAGCGAGGCTGAAACTTTATCTG-3' & 5'-TCACACTCGGCTTGGTCTTTG-3' \\
PRB3 & 5'-CCTCCAGCAAGATGCTACTGATT-3' & 5'-GGGAGATTCTTCCTGGCTGA-3' \\
ZNF717 & 5'-CCTTTCGCTGTAAGTCATTCCT-3' & 5'-TCAGAGAACTCATGCTGGCA-3' \\
MAST4 & 5'-CTTGAACTCTGCCTCAAGCATTC-3' & 5'-ACAAGAACTGGTTTGGTAC-3' \\
SPANXN2 & 5'-GAGGAGGACGAAGGCCTAGA-3' & 5'-CTCACTACCAATGGCGATGA-3' \\
TRIOBP & 5'-CCAGGCTTCCTCCATGACAC-3' & 5'-TGTGTCCAGCAGGACGATC-3' \\
GAPDH & 5'-TGAAGGGCATTCTGGGATAC-3' & 5'-TGTGGACACCACCTGTAGGA-3'
\end{tabular}

DPCR1, diffuse panbronchiolitis critical region 1; DSPP, dentin sialophospho protein; MGAM, maltase-glucoamylase ( $\alpha$-glucosidase); EGFL7, EGF like domain, multiple 7; MX2 myxovirus (influenza virus) resistance; LRRC50, dynein, axonemal, assembly factor 1; PRDM2, PR domain containing 2, with ZNF domain, RIZ1; PRDM8, PR domain containing 8; LRP1B, low density lipoprotein receptor-related protein 1B; RP1L1, retinitis pigmentosa 1-like 1; PRB3, proline-rich protein BstNI subfamily 3; ZNF717, zinc finger protein 717; MAST4, microtubule associated serine/threonine kinase; SPANXN2, SPANX family member N2; TRIOBP, TRIO and F-actin binding protein; GAPDH, glyceraldehyde 3-phosphate dehydrogenase.

(dbSNP) version 130, which is maintained by the National Center for Biotechnology Information, were excluded.

Mutation validation. Primer3 software (version 0.4.0; http://frodo.wi.mit.edu/primer3/) was used to generate primers for the PCR amplification of variants identified via exome sequencing or exons covered in additional screening using a SOLiD5500xl sequencer (Thermo Fisher Scientific, Inc.; Table II). The DNA ladder (DL1000; Takara Bio, Inc., Otsu, Japan) and ethidium bromide were purchased from Takara Bio, Inc. Amplification products of an appropriate size were identified using agarose gel electrophoresis (100 V, $30 \mathrm{~min})$. Amplicons from 3 normal pituitary and 28 pituitary tumor DNA molecules coupled with leukocyte were sequenced using forward and reverse primers. Variants were confirmed by at least two independent sequences from various primers.

$R N A$ extraction and reverse transcription-quantitative $P C R$ $(R T-q P C R)$. Total RNA was extracted from frozen normal pituitary and pituitary adenomas $(\sim 50 \mathrm{mg})$ using the TRIzol Reagent (Invitrogen; Thermo Fisher Scientific, Inc.). RT-qPCR was performed as described previously (23), using the Applied Biosystems 7500 Fast System (Thermo Fisher Scientific, Inc.) and the primers indicated in Table III. The fold-change in differential expression for each gene was calculated using the comparative $\mathrm{Cq}$ method (also known as the $2^{-\Delta \Delta \mathrm{Cq}}$ method), as previously described (24).

Immunohistochemical analysis. Pituitary adenomas and pituitary gland specimens were sectioned to a thickness of $5 \mu \mathrm{m}$ in paraffin wax (Leica Biosystems Richmond Inc.). The sections were subjected to gradient dewaxing, removed of water, treated with fresh $3 \%$ hydrogen peroxide (ZSGB-BIO) at room temperature for $10 \mathrm{~min}$ and washed with phosphate-buffered saline ( $\mathrm{pH}$ 7.2; ZSGB-BIO) 3 times for 5 min each. For microwave repair, the specimens were placed in $0.01 \%$ citric acid (pH 6.0; ZSGB-BIO), kept warm in a microwave oven (600 W; LG Electronics Appliances Co., Ltd., Tianjin, China) for $10 \mathrm{~min}$, allowed to cool to room temperature and washed once with PBS for $10 \mathrm{~min}$. Antibody repair solution was added at room temperature for $10 \mathrm{~min}$, and then washed 3 times with PBS for 5 min each time. PRDM2 antibody (monoclonal; Abcam Inc., Eugene, OR, USA) was added at a 1:200 dilution and incubated at $4^{\circ} \mathrm{C}$ overnight. The DyLight ${ }^{\mathrm{TM}}$-conjugated AffiniPure secondary antibody with fluorescence (ZSGB-BIO) was added at room temperature for $1 \mathrm{~h}$, followed by 3 washes with PBS for 5 min each time. Streptavidin-biotin complex was added for $20 \mathrm{~min}$ and then washed with PBS for $5 \mathrm{~min}$. Sections were mounted with Prolong Gold Antifade reagent with 4',6-diamidino-2-phenylindole (DAPI; Invitrogen; Thermo Fisher Scientific, Inc.). Sections were analyzed with a LEICA-TCS-SP5II (Leica Microsystems GmbH, Wetzlar, Germany) to estimate the percentage of DAPI-stained cells displaying PRDM2 immunoreactivity.

Statistical analysis. All statistical analyses were performed using SPSS version 20.0 (IBM SPSS, Armonk, NY, USA). For comparisons, one-way analyses of variance, $\chi^{2}$ tests, Wilcoxon rank-sum tests and two-tailed Student's $t$-tests were performed as appropriate. Binary logistic regression was performed 


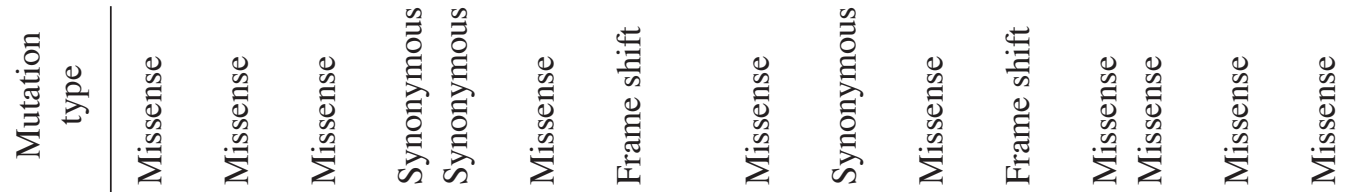

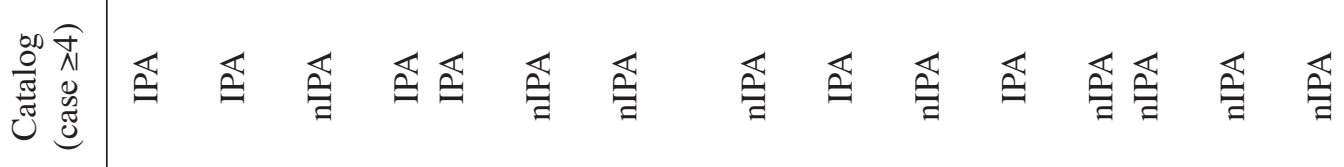

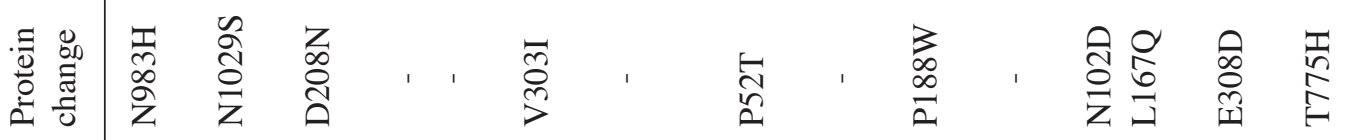

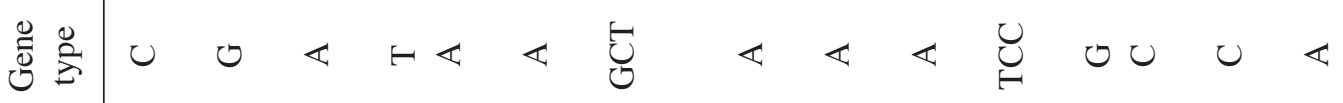

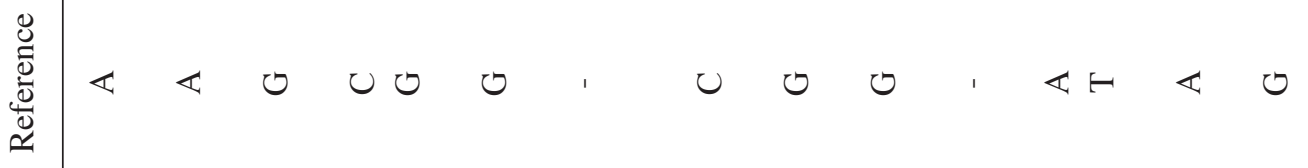

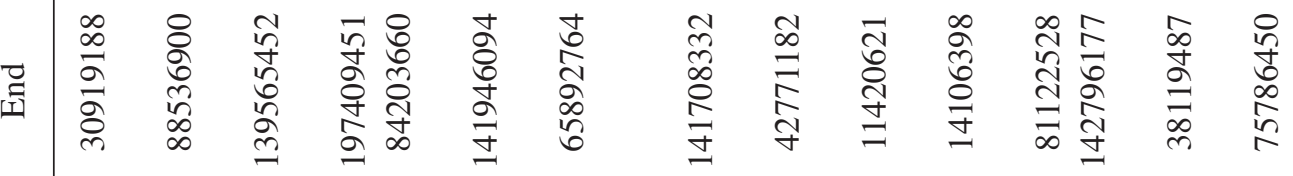

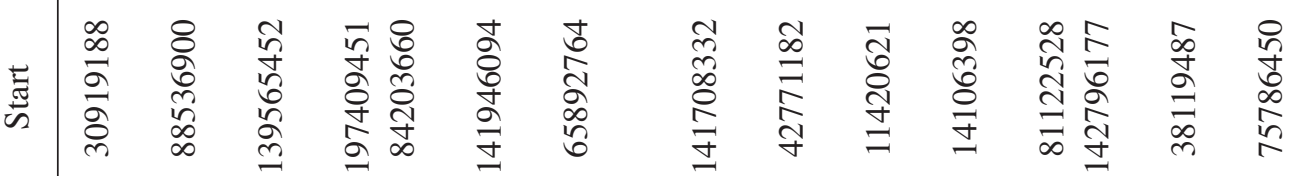

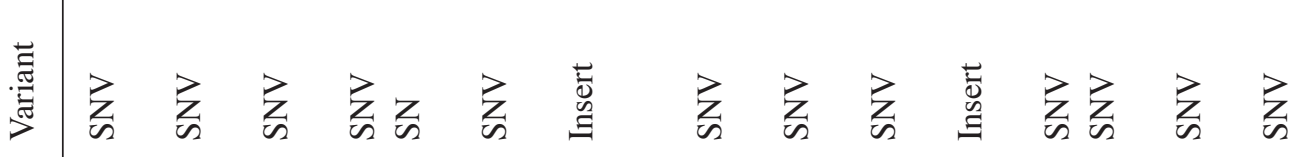
造

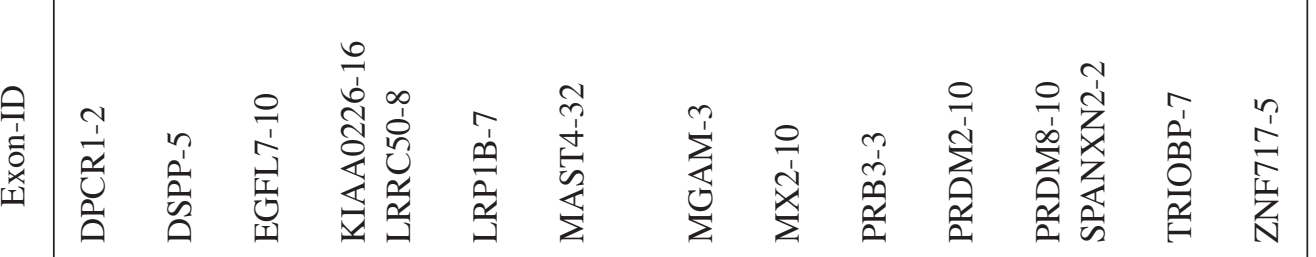

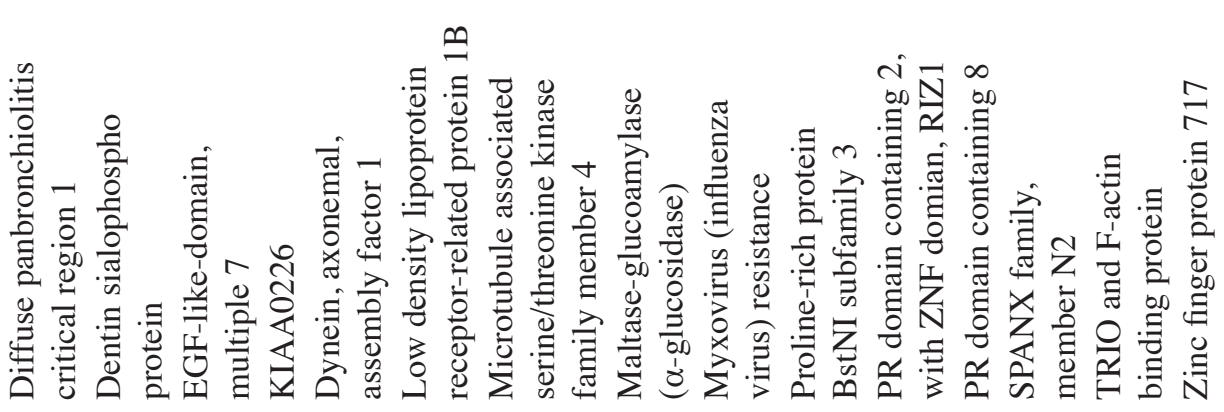


A
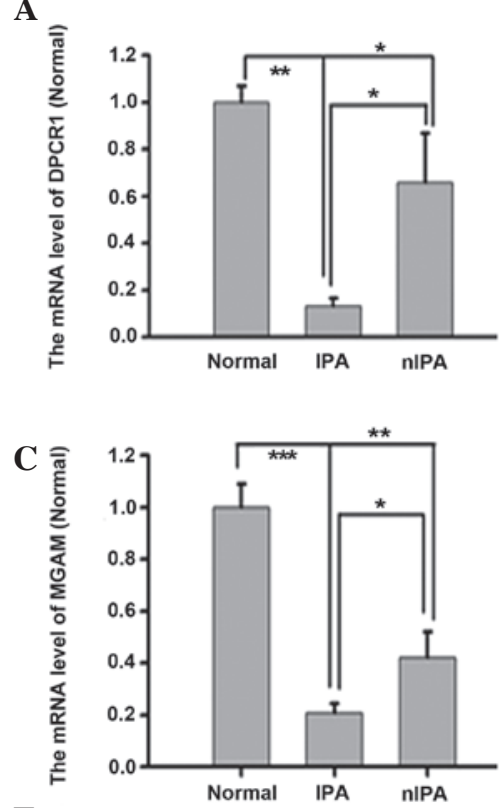

E

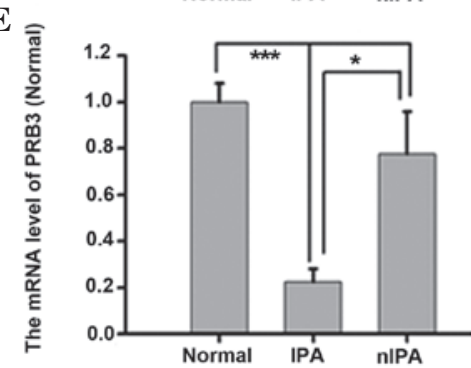

G

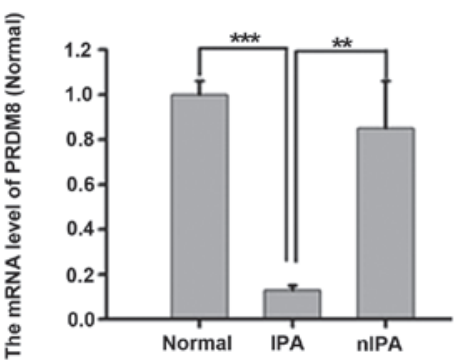

I

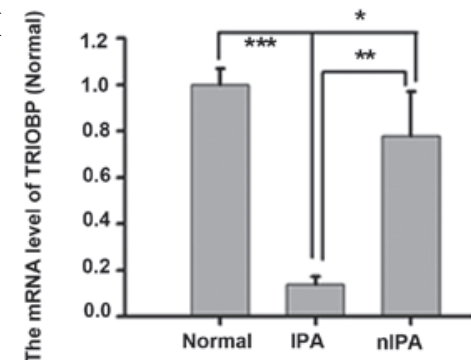

$\mathbf{B}$

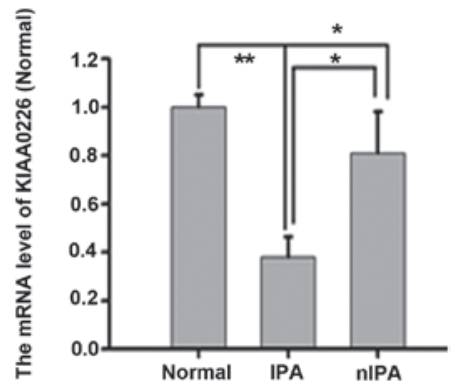

D

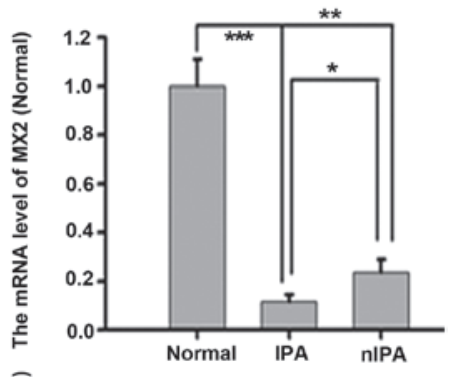

$\mathrm{F}$ สิ

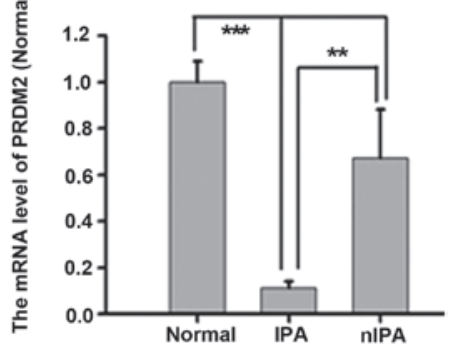

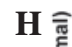
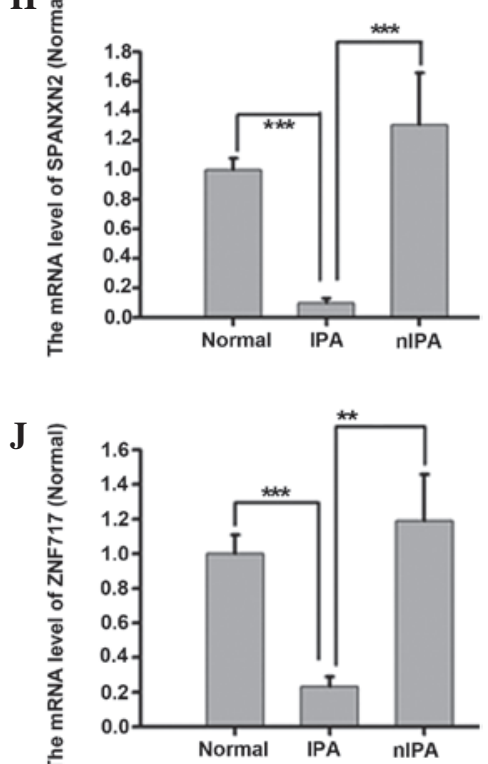

Figure 1. mRNA levels of (A) DPCR1, (B) KIAA0226, (C) MGAM, (D) MX2, (E) PRB3, (F) PRDM2, (G) PRDM8, (H) SPANXN2, (I) TRIOBP and (J) ZNF717 with variants, which are lower in IPA specimens compared with nIPA specimens. mRNA levels of DPCR1, KIAA0226, MX2, PRB3, PRDM2, PRDM8, SPANXN2, TRIOBP and ZNF717 in IPA specimens were 50\% decreased compared with nIPA specimens. In particular, DPCR1, PRDM2, PRDM8, SPANXN2 and ZNF717 mRNA levels in IPA specimens were approximately four-fold lower compared with nIPA specimens $(\mathrm{P}<0.01)$. $\mathrm{n}=3-8$. Groups were Normal, IPA and nIPA. nIPA, non-invasive pituitary adenoma; IPA, invasive pituitary adenoma; Normal, normal pituitary; mRNA, messenger RNA; DPCR1, diffuse panbronchiolitis critical region 1; MX2, myxovirus (influenza virus) resistance; PRB3, proline-rich protein BstNI subfamily 3; PRDM2, PR domain containing 2, with ZNF domain, RIZ1; PRDM8, PR domain containing 8; SPANXN2, SPANX family member N2; TRIOBP, TRIO and F-actin binding protein; ZNF717, zinc finger protein 717. ${ }^{*} \mathrm{p}<0.05 ;{ }^{* *} \mathrm{p}<0.01 ;{ }^{* * *} \mathrm{p}<0.001$.

to identify the independent factors associated with pituitary adenoma recurrence. $\mathrm{P}<0.05$ was used to indicate a statistically significant difference.

\section{Results}

Identification of variant genes by whole-exome sequencing. 
For the identification of tumor-specific somatic variants, whole-exome capture using DNA from the discovery set of six IPAs and six nIPAs yielded excellent target region coverage, with $\sim 72 \%$ of the exome covered to a depth of at least 30 -fold between the somatic variant calling algorithm and confirmatory sequencing. Several prioritization steps were taken to decrease the number of genetic variants and to find the potentially pathogenic variants, as follows: i) Variants should have a deleterious effect on protein function (as predicted by protein prediction software, such as PolyPhen-2, MutationTaster and SIFT); ii) variants should be present at sufficient allele frequency to represent likely heterozygous or homozygous changes (i.e., present from early in the tumorigenic process), although deviation from the expected heterozygous or homozygous allele frequencies may represent either contamination with normal tissue or the preference of the sequence and alignment process for the wild-type allele, as previously reported $(25,26)$; and iii) variants should be involved in biological processes relevant to tumorigenesis (27). Approximately $90 \%$ of single-nucleotide variants (SNVs) resulted in missense amino-acid changes, whereas the remaining $(\sim 10 \%)$ were synonymous changes. Over $70 \%$ of the SNVs occurred as C:G-T:A transitions, and $<30 \%$ were transversions. Using stringent variant calling and filtering parameters (16), 233 variants were identified in the specimens.

In addition, five variants (C8orf79, chr8:12879694; FSHD region gene 1 family member $\mathrm{B}$, pseudogene, chr20:29632674; mucin 2, oligomeric mucus/gel-forming, chr11:1092715; mucin 6, oligomeric mucus/gel-forming, chr11:1018092; and solute carrier family 5 member 3 , chr21:35467473) were present in all specimens, and 47 were detected in either the IPA or nIPA. Of these, 15 were somatic variants confirmed by dideoxynucleotide sequencing. Of the 15 confirmed variants, 13 occurred as SNVs, including three synonymous SNVs, and two comprised insertions (Table IV). The genes with variants were generally associated with angiogenesis, metabolism, cell cycle phase, cellular component organization, cytoskeleton and biological immunity at a cellular level. The genes include: EGF like domain, multiple 7 (EGFL7), associated with angiogenesis; low density lipoprotein receptor-related protein 1B (LRP1B) and maltase-glucoamylase ( $\alpha$-glucosidase) associated with cell metabolism; dentin sialophospho protein (DSPP), PR domain containing 2, with ZNF domain, RIZ1 (PRDM2), PR domain containing 8 (PRDM8) and zinc finger protein 717 (ZNF717) associated with cell proliferation; dynein, axonemal, assembly factor 1 (LRRC50), microtubule associated serine/threonine kinase and TRIO and F-actin binding protein (TRIOBP) associated with cytoskeleton; myxovirus (influenza virus) resistance (MX2) associated with cell cycle phase; diffuse panbronchiolitis critical region 1 (DPCR1), proline-rich protein BstNI subfamily 3 (PRB3) and SPANX family member N2 (SPANXN2) associated with immune response; and KIAA0226 associated with vesicle trafficking.

Furthermore, three variants were indicated in PRDM2 in five separate IPA specimens, including two synonymous and one frame shift. One mutation was indicated in PRDM8 (missense, N102D) and PR domain containing 10 (missense, S1018R) in a tumor separately (data not shown), and four variants (R246Q, G272C, S501F and A1247G) were indicated in PR domain containing 16 in three separate IPA specimens (data not shown).
Table V. Association between PRDM2 mRNA level in pituitary adenomas and various clinical parameters.

\begin{tabular}{|c|c|c|c|c|}
\hline \multirow[b]{2}{*}{ Parameter } & \multicolumn{2}{|c|}{ mRNA level } & \multirow[b]{2}{*}{$\chi^{2}$} & \multirow[b]{2}{*}{ P-value } \\
\hline & $\mathrm{High}^{\mathrm{a}}$ & Low & & \\
\hline All cases & 12 & 12 & & \\
\hline Age, years & & & 0.689 & 0.406 \\
\hline$\geq 50$ & 4 & 6 & & \\
\hline$<50$ & 8 & 6 & & \\
\hline Gender & & & 0.168 & 0.682 \\
\hline Male & 6 & 7 & & \\
\hline Female & 6 & 5 & & \\
\hline Tumor size, $\mathrm{cm}$ & & & 0.178 & 0.673 \\
\hline$\geq 2$ & 5 & 4 & & \\
\hline$<2$ & 7 & 7 & & \\
\hline Recurrence $^{\mathrm{b}}$ & & & 6.511 & 0.011 \\
\hline Yes & 4 & 10 & & \\
\hline No & 8 & 2 & & \\
\hline
\end{tabular}

${ }^{a}$ Median expression level was used as the cutoff between the high and low mRNA levels. ${ }^{b}$ Recurrence was defined as the discovery of an elevated PRL level at any time in the postoperative surveillance period after an initial remission. Low PRDM2 mRNA levels were defined as values below the 50th percentile of the 12 patients; values at or above 50th percentile were classified as high levels. PRDM2, PR domain containing 2, with ZNF domain, RIZ1; mRNA, messenger RNA.
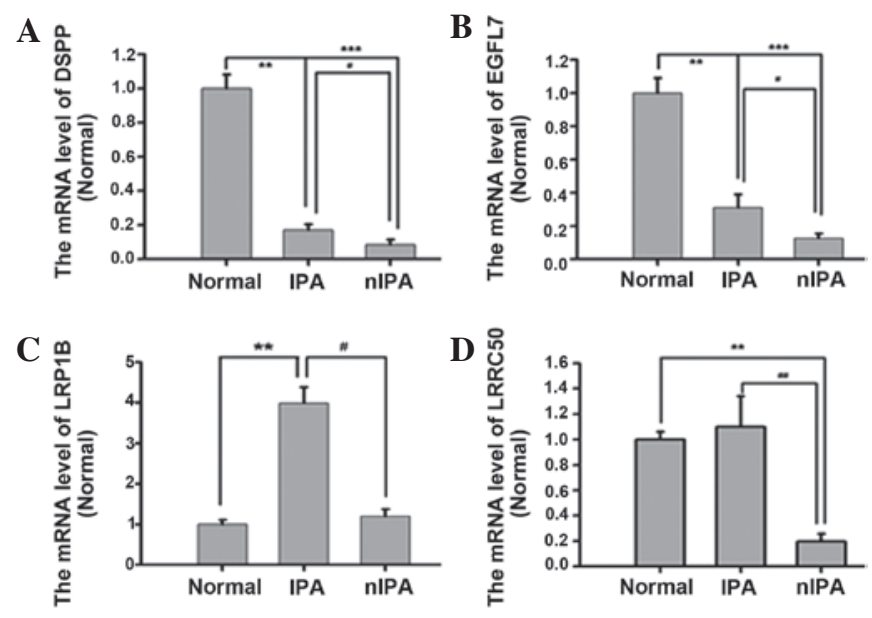

Figure 2. mRNA level of (A) DSPP, (B) EGFL7, (C) LRP1B and (D) LRRC50 with variants, which are increased in IPA specimens compared with nIPA specimens. mRNA levels of DSPP, EGFL7 and LRRC50 were increased in IPA compared with in nIPA specimens $(\mathrm{P}<0.01) . \mathrm{n}=3-8$. Groups were Normal, nIPA and IPA. mRNA, messenger RNA; Normal, normal pituitary; nIPA, non-invasive pituitary adenoma; IPA, invasive pituitary adenoma; DSPP, dentin sialophospho protein; EGFL7, EGF like domain, multiple 7; LRRC50, dynein, axonemal, assembly factor $1 .{ }^{*} \mathrm{p}<0.05 ;{ }^{* *} \mathrm{p}<0.01 ;{ }^{* * *} \mathrm{p}<0.001$.

Analysis of the expression of variant genes by RT-qPCR. RT-qPCR was used to test whether the invasion of pituitary adenomas was associated with differences in the expression levels of 15 genes. Expression of DPCR1, KIAA0226, MX2, 
A

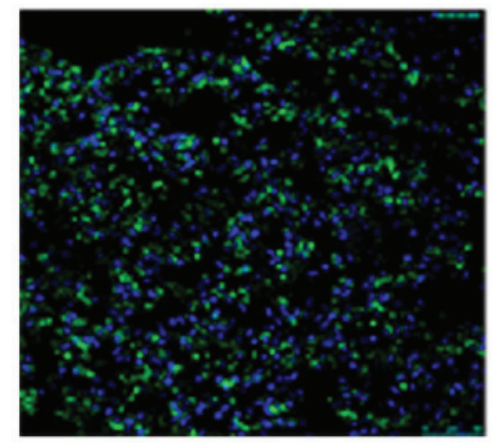

B

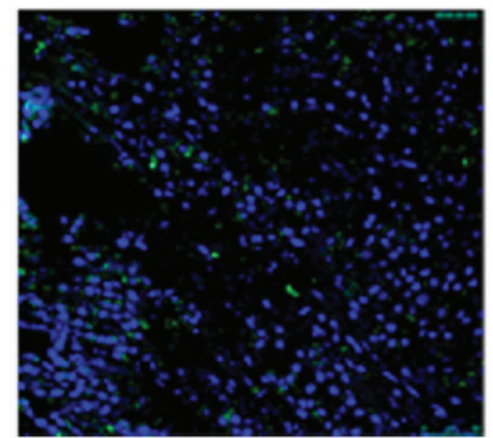

C

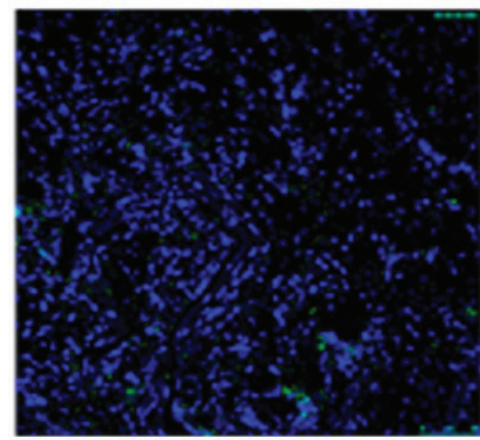

Figure 3. Confocal images show the number of PRDM2-positive puncta in recurrent pituitary adenomas. (A) Normal pituitary; (B) non-invasive pituitary adenoma; (C) inviasive pituitary adenoma. Green, PRDM2; dilution, 1:200. Blue, 4',6-diamidino-2-phenylindole; dilution, 1:3,000. Scale bar, $50 \mu \mathrm{m}$. PRDM2, PR domain containing 2, with ZNF domain, RIZ1.

PRB3, PRDM2, PRDM8, SPANXN2, TRIOBP and ZNF717 in IPA specimens was $50 \%$ decreased compared with in nIPA specimens (Fig. 1). In particular, DPCR1, PRDM2, PRDM8, SPANXN2 and ZNF717 mRNA levels in IPA specimens were approximately four-fold decreased compared with in nIPA specimens ( $\mathrm{P}=0.003,0.007,0.009$ and 0.004 , respectively). By contrast, the mRNA levels of DSPP, EGFL7, LRP1B and LRRC50 in IPA specimens were increased compared with in nIPA specimens $(\mathrm{P}=0.041,0.037,0.022$ and 0.013 , respectively; Fig. 2).

PRDM2 levels are associated with recurrence in pituitary adenomas. The usual morphological signs of tumor aggression are poorly associated with the invasive potential of pituitary tumors, proliferation capacity, tendency of post-surgical recurrence and global biological behavior (28). PRDM2 contains a PR domain that demonstrates histone $\mathrm{H} 3$ lysine 9 methylation activity (29). Therefore, whether decreased levels of PRDM family mRNA in pituitary adenomas was associated with certain clinical parameters was assessed (Table V). No significant association was indicated between PRDM2 mRNA levels and age, gender or tumor size. However, decreased PRDM2 protein levels were more frequently observed in recurrent tumors (Fig. 3). Furthermore, binary multivariate regression revealed that decreased levels of PRDM2 were independently associated with tumor recurrence (odds ratio, $0.065 ; 95 \%$ confidence interval, 0.050-0.832; $\mathrm{P}=0.036)$.

\section{Discussion}

Tumor invasion may be based on clinical, radiological and pathological features (2-6,8-21,23,27-30). However, no standard or comparable score on IPA is generally accepted, except for radiological classification. IPAs are associated with a poor prognosis, as therapeutic options are limited. In addition, IPAs tend to recur quickly following initial treatment, are generally unresponsive to therapy and are a challenge to manage (5). In the present study, 15 somatic variants that are mainly associated with metabolism, cell cycle phase, cellular component organization, cytoskeleton and biological immunity at a cellular level, but not with genes previously implicated in pituitary adenomas, were identified by whole-exome sequencing.

A growing body of evidence suggests a coevolutionary model of cancer, wherein the cross-talk between tumor cells and the host determine the malignant potential of individual tumors (31). Endogenous T cells respond to and infiltrate tumors, significantly delaying malignant progression in mouse models (32). Low expression levels of interleukin- 6 and signal transducer and activator of transcription 3 were indicated to be significant in the dysimmunity of pituitary adenoma (33). DPCR1, located between major histocompatibility complex (MHC), class I (HLA)-B and HLA-A on chromosome 6p21.33, is classified as one of the HLA molecules. The DPCR1 gene may contain markers for diagnosis of diffuse pan-bronchiolitis, a bronchiolar disease that affects human airways (34). To fully escape the immune system, cancer cells typically mutate to decrease the expression of antigens, lose expression of HLA proteins or employ an aberrant antigen processing pathway (35). However, to the best of our knowledge, the association between DPCR1 variations and the risk of IPA has not yet been investigated. The mRNA level of DPCR1 is approximately four-fold lower in IPA compared with in nIPA specimens; therefore, the invasion of pituitary adenomas is hypothesized to be associated with the induction of immune-escape via the downregulation of DPCR1.

The expansion of solid tumors depends on the continuous growth of novel blood vessels from pre-existing capillaries. The role of angiogenesis and tumor blood vessels in the pathogenesis of pituitary tumors remains a mystery. A previous study indicated the involvement of prolactin during vasculature remodelling by acting on the endothelial and perivascular cells in pituitary adenomas (36). The vascular-specific secreted factor EGFL7 is a component of the interstitial extracellular matrix (ECM) and regulates the proper spatial organization of endothelial cells within each filopodia, affecting the collective movement of the cells (37). A previous study indicated that the expression of EGFL7 in neural stem cells (NSCs) in vitro decreased NOTCH-specific signaling and resulted in the decreased proliferation and self-renewal of NSCs (38). EGFL7 acts as a soluble NOTCH inhibitor, which is in contrast to the typical $\mathrm{NOTCH}$ 
inhibitory molecules that are expressed by adjacent cells as transmembrane proteins $(39,40)$. A previous study indicated that the differentially expressed genes involved in this pathway were delta-like 1 homolog, C-terminal binding protein 2, hes family bHLH transcription factor (HES)1, HES5 and E1A binding protein p300 in plurihormonal pituitary adenomas (41). Another study used RT-qPCR assays and western blot analyses to observe upregulated NOTCH3 and jagged 1 (JAG1) in human NFPAs; furthermore, NOTCH3 was positively associated with JAG1 at the mRNA and protein levels (42). The elevated expression of EGFL7 in IPAs may be associated with invasive behavior via activation of the NOTCH pathway.

The attachment, movement and invasion of cancer cells are facilitated by the actin cytoskeleton and tubulin, as the structural element of microtubules $(8-21,23,27-43)$. ECM proteins send information to the cells, which respond with a specific cytoskeletal organization that modulates specific cellular patterns of behavior in GH3 tumor pituitary cell (9-21,23,27-44). Pituitary tumor cells acquire different patterns: mesenchymal, and leucocyte/amoeboid, the last observed in the invasive adenomas and amoeboid migration pattern has been associated with high invasion capacity (10-21,23,27-45). LRRC50 was identified as a putative ciliary protein in two independent bioinformatic studies $(46,47)$. LRRC50 hu255H mutants develop pronephric cysts with an increased proliferative index, severely reduced brush border, and disorganized pronephric cilia manifesting impaired localized fluid flow consistent with ciliary dysfunction (48). LRRC50 to be a novel tumor suppressor implicated in human seminoma pathogenesis. A pathogenic Gln307Glu change is significantly enriched in individuals with seminoma tumors (48). The Arg488Glu mutation of LRRC50 in IPA is, therefore, hypothesized to be a loss-of-function mutation due to the increased mRNA levels in IPAs compared with in nIPAs.

Increasing evidence has demonstrated the primary roles of tumor suppressors, oncogenes and cell cycle abnormalities in pituitary tumorigenesis. The PRDM group of proteins is an evolutionarily conserved protein family with 17 predicted members in humans; of which, few protein members have been characterized (49). Numerous studies suggest that PRDM family proteins interact with a number of chromatin modifying proteins, and act primarily as negative regulators of transcription (50-54). PRDM2/RIZ is a binding partner for the retinoblastoma tumor suppressor protein and is the frequent target for inactivation in a variety of human tumors, including breast, liver, and colon cancers (55). Its tumor suppressor function is directly confirmed by the tumorigenic phenotype of mice deficient for RIZ1, the PR-containing isoform of PRDM2. In the present study, PRDM2 and PRDM8 mRNA levels were approximately five-fold lower in IPA specimens compared with nIPA specimens $(\mathrm{P}=0.007$ and 0.009 , respectively). In addition, binary multivariate regression revealed that decreased levels of PRDM2 were independently associated with tumor recurrence.

Exome sequencing studies allow the comprehensive testing of coding variation in an unbiased manner. The results of the current study demonstrate that whole-exome sequencing will be particularly valuable for the identification of genes under conditions in which mapping has been confounded by locus heterogeneity and uncertainty of the boundaries of diagnostic classification. Whole-exome sequencing may be useful in the future for a wide range of applications to medicine.

\section{Acknowledgements}

The present study was supported by the Research Special Fund for Public Welfare Industry of Health of China (grant no. 201402008), the National Natural Science Foundation of China (grant no. 81272522) and Beijing Natural Science Foundation of China (grant no. 7162035).

\section{References}

1. Ezzat S, Asa SL, Couldwell WT, Barr CE, Dodge WE, Vance ML and McCutcheon IE: The prevalence of pituitary adenomas: A systematic review. Cancer 101: 613-619, 2004.

2. Bronstein MD and Melmed S: Pituitary tumorigenesis. Arq Bras Endocrinol Metabol 49: 615-625, 2005 (In Portuguese).

3. Al-Shraim M and Asa SL: The 2004 World Health Organization classification of pituitary tumors: What is new? Acta Neuropathol 111: 1-7, 2006.

4. Scheithauer BW, Kovacs KT, LawsER Jr and Randall RV: Pathology of invasive pituitary tumors with special reference to functional classification. Journal Neurosur 65: 733-744, 1986.

5. Colao A, Grasso LF, Pivonello R and Lombardi G: Therapy of aggressive pituitary tumors. Expert Opin Pharmacother 12: 1561-1570, 2011.

6. Di Ieva A, Rotondo F, Syro LV, Cusimano MD and Kovacs K: Aggressive pituitary adenomas-diagnosis and emerging treatments. Nat Rev Endocrinol 10: 423-435, 2014.

7. Lloyd RV, Kovacs K, Young Jr WF, Farrell WE, Asa SL, Trouillas J, Kontogeorgos G, Sano T, Scheithauer BW and Horvath E: Tumors of the pituitary. In: DeLellis RA, Lloyd RV, Heitz PU and Eng C (eds.): World Health Organization Classification of Tumors: Pathology and Genetics: Tumors of Endocrine Organs. IARC Press, Lyon, pp10-47, 2004.

8. Gejman R, Swearingen B and Hedley-Whyte ET: Role of Ki-67 proliferation index and p53expression in predicting progression of pituitary adenomas. Hum Pathol 39: 758-766, 2008

9. Zada G, Woodmansee WW, Ramkissoon S, Amadio J, Nose V and Laws ER Jr: Atypical pituitary adenomas: Incidence, clinical characteristics, and implications. J Neurosurg 114: 336-344, 2011.

10. Mete O, Ezzat S and Asa SL: Biomarkers of aggressive pituitary adenomas. J Mol Endocrinol 49: R69-R78, 2012.

11. Melmed S: 2004 World Health Organization classification of pituitary tumors: What is new? Acta Neuropathol 111: 78-79, 2006.

12. Righi A, Morandi L, Leonardi E, Farnedi A, Marucci G, Sisto A, Frank G, Faustini-Fustini M, Zoli M, Mazzatenta D, et al: Galectin-3 expression in pituitary adenomas as a marker of aggressive behavior. Hum Pathol 44: 2400-2409, 2013.

13. Galland F, Lacroix L, Saulnier P, Dessen P, Meduri G, Bernier M, Gaillard S, Guibourdenche J, Fournier T, Evain-Brion D, et al: Differential gene expression profiles of invasive and non-invasive non-functioning pituitary adenomas based on microarray analysis. Endocr Relat Cancer 17: 361-371, 2010.

14. Schvartzman JM, Sotillo R and Benezra R: Mitotic chromosomal instability and cancer: Mouse modelling of the human disease. Nat Rev Cancer 10: 102-115, 2010.

15. Pilarski R and Nagy R: Genetic testing by cancer site: Endocrine system. Cancer J 18: 364-371, 2012.

16. Newey PJ, Nesbit MA, Rimmer AJ, Head RA, Gorvin CM, Attar M, Gregory L, Wass JA, Buck D, Karavitaki N, et al: Whole-exome sequencing studies of nonfunctioning pituitary adenomas. J Clin Endocrinol Metab 98: E796-E800, 2013.

17. Asa SL and Ezzat S: The pathogenesis of pituitary tumours. Nat Rev Cancer 2: 836-849, 2002.

18. Zhan X, Desiderio DM, Wang X, Zhan X, Guo T, Li M, Peng F, Chen X, Yang H, Zhang P, et al: Identification of the proteomic variations of invasive relative to non-invasive nonfunctional pituitary adenomas. Electrophoresis 35: 2184-2194, 2014.

19. Yoshida K, Sanada M, Shiraishi Y, Nowak D, Nagata Y, Yamamoto R, Sato Y, Sato-Otsubo A, Kon A, Nagasaki M, et al: Frequent pathway mutations of splicing machinery in myelodysplasia. Nature 478: 64-69, 2011. 
20. Agrawal N, Frederick MJ, Pickering CR, Bettegowda C, Chang K, Li RJ, Fakhry C, Xie TX, Zhang J, Wang J, et al: Exome sequencing of head and neck squamous cell carcinoma reveals inactivating mutations in NOTCH1. Science 333: 1154-1157, 2011.

21. Ni X, Zhuo M, Su Z, Duan J, Gao Y, Wang Z, Zong C, Bai H, Chapman AR, Zhao J, et al: Reproducible copy number variation patterns among single circulating tumor cells of lung cancer patients. Proc Natl Acad Sci USA 110: 21083-21088, 2013.

22. Enseñat J, Ortega A, Topcewski T, Vilalta J, Obiols G, Mesa J and Sahuquillo J: Predictive value of the Knosp classification in grading the surgical resection of invasive pituitary macroadenomas. A prospective study of 23 cases. Neurocirugia (Astur) 17: 519-26, 2006 (In Spanish).

23. Wang F, Gao H, Li C, Bai J, Lu R, Cao L, Wu Y, Hong L, Wu Y, Lan X and Zhang Y: Low levels of PRB3 mRNA are associated with dopamine-agonist resistance and tumor recurrence in prolactinomas. J Neurooncol 116: 83-88, 2014.

24. Livak KJ and Schmittgen TD: Analysis of relative gene expression data using real-time quantitative PCR and the 2(-Delta Delta C(T)) Method. Methods 25: 402-408, 2001.

25. Jiao Y, Shi C, Edil BH, de Wilde RF, Klimstra DS, Maitra A, Schulick RD, Tang LH, Wolfgang CL, Choti MA, et al: DAXX/TRX, MEN1, and mTOR pathway genes are frequently altered in pancreatic neuroendocrine tumors. Science 331 1199-1203, 2011.

26. Newey PJ, Nesbit MA, Rimmer AJ, Attar M, Head RT, Christie PT, Gorvin CM, Stechman M, Gregory L, Mihai R, et al: Whole-exome sequencing studies of nonhereditary (sporadic) parathyroid adenomas. J Clin Endocrinol Metab 97 : E1995-E2005, 2012

27. Barbieri CE, Baca SC, Lawrence MS, Demichelis F, Blattner M, Theurillat JP, White TA, Stojanov P, Van Allen E, Stransky N, et al: Exome sequencing identifies recurrent SPOP, FOXA1 and MED12 mutations in prostate cancer. Nat Genet 44: 685-689, 2012.

28. Popescu MN, Ionescu E, Iovănescu LC, Cotoi BV, Popescu AI, Gănescu AE, Glodeanu A, Geormăneanu C, Moraru A and Pătraşcu A: Clinical aggression of prolactinomas: Correlations with invasion and recurrence. Rom J Morphol Embryol 54: 1075-1080, 2013

29. Varier RA and Timmers HT: Histone lysine methylation and demethylation pathways in cancer. Biochimica Biophysica Acta 1815: 75-89, 2011

30. Tanase C, Ogrezeanu I and Badiu C (eds): Pituitary tumor classification: Functionality, invasiveness and aggressiveness. In: Molecular Pathology of Pituitary Adenomas. Elsevier, Amsterdam, Netherlands, pp1-18, 2011.

31. Dhodapkar MV: Personalized immune-interception of cancer and the battle of two adaptive systems-when is the time right? Cancer Prev Res (Phila) 6: 173-176, 2013.

32. DuPage M, Cheung AF, Mazumdar C, Winslow MM, Bronson R, Schmidt LM, Crowley D, Chen J and Jacks T: Endogenous T cell responses to antigens expressed in lung adenocarcinomas delay malignant tumor progression. Cancer Cell 19: 72-85, 2011.

33. Wang W, Xu Z, Fu L, Liu W and Li X: Pathogenesis analysis of pituitary adenoma based on gene expression profiling. Oncol Lett 8: 2423-2430, 2014.

34. Shen FF, Yue WB, Zhou FY, Pan Y, Zhao XK, Jin Y, Song X, $\mathrm{Li} \mathrm{B}$, Han XN, Tang S, et al: Variations in the MHC region confer risk to esophageal squamous cell carcinoma on the subjects from high-incidence area in northern China. PloS One 9: e90438, 2014.

35. Lee JS, Bae JS, Kim JH, Kim JY, Park TJ, Pasaje CF, Park BL, Cheong HS, Uh ST, Park JS, et al: Effect of diffuse panbronchiolitis critical region 1 polymorphisms on the risk of aspirin-exacerbated respiratory disease in Korean asthmatics. Respir Care 57: 758-763, 2012.

36. Osamura RY, Kajiya H, Takei M, Egashira N, Tobita M, Takekoshi S and Teramoto A: Pathology of the human pituitary adenomas. Histochem Cell Biol 130: 495-507, 2008.

37. Schmidt M, Paes K, De Mazière A, Smyczek T, Yang S Gray A, French D, Kasman I, Klumperman J, Rice DS and Ye W: EGFL7 regulates the collective migration of endothelial cells by restricting their spatial distribution. Development 134: 2913-2923, 2007.
38. Schmidt MH, Bicker F, Nikolic I, Meister J, Babuke T, Picuric S, Müller-Esterl W, Plate KH and Dikic I: Epidermal growth factor-like domain 7 (EGFL7) modulates Notch signalling and affects neural stem cell renewal. Nat Cell Biol 11: 873-880, 2009.

39. Bambino K, Lacko LA, Hajjar KA and Stuhlmann H: Epidermal growth factor-like domain 7 is a marker of the endothelial lineage and active angiogenesis. Genesis 52: 657-670, 2014.

40. Louvi A and Artavanis-Tsakonas S: Notch signalling in vertebrate neural development. Nat Rev Neurosci 7: 93-102, 2006.

41. Jiang Z, Gui S and Zhang Y: Analysis of differential gene expression in plurihormonal pituitary adenomas using bead-based fiber-optic arrays. J Neurooncol 108: 341-348, 2012.

42. Lu R, Gao H, Wang H, Cao L, Bai J and Zhang Y: Overexpression of the Notch 3 receptor and its ligand Jagged 1 in human clinically non-functioning pituitary adenomas. Oncol Lett 5: 845-851, 2013.

43. Howard J and Hyman AA: Dynamics and mechanics of the microtubule plus end. Nature 422: 753-758, 2003.

44. Azorín E, Romero-Pérez B, Solano-Agama C, de la Vega MT, Toriz CG, Reyes-Márquez B, González-Pozos S, Rosales-García VH, Del Pliego MG, Sabanero M and Mendoza-Garrido ME: GH3 tumor pituitary cell cytoskeleton and plasma membrane arrangement are determined by extracellular matrix proteins: Implications on motility, proliferation and hormone secretion. Int J Physiol Pathophysiol Pharmacol 6: 66-83, 2014.

45. del Pliego MG, Aguirre-Benítez E, Paisano-Cerón K, Valdovinos-Ramírez I, Rangel-Morales C, Rodríguez-Mata V, Solano-Agama C, Martín-Tapia D, de la Vega MT, Saldoval-Balanzario M, et al: Expression of Eag1 K+ channel and ErbBs in human pituitary adenomas: Cytoskeleton arrangement patterns in cultured cells. Int J Clin Exp Pathol 6: 458-468, 2013.

46. Stolc V, Samanta MP, Tongprasit W and Marshall WF: Genome-wide transcriptional analysis of flagellar regeneration in Chlamydomonas reinhardtii identifies orthologs of ciliary disease genes. Proc Natl Acad Sci USA 102: 3703-3707, 2005.

47. Avidor-Reiss T, Maer AM, Koundakjian E, Polyanovsky A, Keil T, Subramaniam S and Zuker CS: Decoding cilia function: Defining specialized genes required for compartmentalized cilia biogenesis. Cell 117: 527-539, 2004.

48. Van Rooijen E, Giles RH, Voest EE, van Rooijen C, Schulte-Merker S and van Eeden FJ: LRRC50, a conserved ciliary protein implicated in polycystic kidney disease. J Am Soc Nephrol 19: 1128-1138, 2008.

49. Basten SG, Davis EE, Gillis AJ, van Rooijen E, Stoop H, Babala N, Logister I, Heath ZG, Jonges TN, Katsanis N, et al: Mutations in LRRC50 predispose zebrafish and humans to seminomas. PLoS Genet 9: e1003384, 2013.

50. Hohenauer T and Moore AW: The Prdm family: Expanding roles in stem cells and development. Development 139: 2267-2282, 2012.

51. Davis CA, Haberland M, Arnold MA, Sutherland LB, McDonald OG, Richardson JA, Childs G, Harris S, Owens GK and Olson EN: PRISM/PRDM6, a transcriptional repressor that promotes the proliferative gene program in smooth muscle cells. Mol Cell Biol 26: 2626-2636, 2006.

52. Kajimura S, Seale P, Tomaru T, Erdjument-Bromage H, Cooper MP, Ruas JL, Chin S, Tempst P, Lazar MA and Spiegelman BM: Regulation of the brown and white fat gene programs through a PRDM16/CtBP transcriptional complex. Genes Dev 22: 1397-1409, 2008

53. Izutsu K, Kurokawa M, Imai Y, Maki K, Mitani K and Hirai H: The corepressor CtBP interacts with Evi-1 to repress transforming growth factor beta signaling. Blood 97: 2815-2822, 2001.

54. Yu J, Angelin-Duclos C, Greenwood J, Liao J and Calame K: Transcriptional repression by blimp-1 (PRDI-BF1) involves recruitment of histone deacetylase. Mol Cell Biol 20: 2592-2603, 2000.

55. Tam W, Gomez M, Chadburn A, Lee JW, Chan WC and Knowles DM: Mutational analysis of PRDM1 indicates a tumor-suppressor role in diffuse large B-cell lymphomas. Blood 107: 4090-4100, 2006 